\title{
DISTRIBUTION OF RESONANCES FOR SPHERICAL BLACK HOLES
}

\section{Antônio SÁ Barreto And Maciej Zworski}

\section{Introduction and statement of results}

The purpose of this note is to apply the methods of geometric scattering theory developed by Briet-Combes-Duclos [6], Gérard-Sjöstrand [14], MazzeoMelrose [22] and the second author [30] in the simplest model of a Black Hole: the De Sitter-Schwarzschild metric. We show that the resonances (or the quasi normal modes, in the terminology of Chandrasekhar [8]) are globally defined in $\mathbb{C}$ and that in any strip below the real axis and for large angular momenta, $l$, they are well approximated by the "pseudo-poles"

$$
\left( \pm l \pm \frac{1}{2}-i\left(k+\frac{1}{2}\right)\right) \frac{\left(1-9 \Lambda m^{2}\right)^{\frac{1}{2}}}{3^{\frac{3}{2}} m}
$$

where $l=1,2, \cdots, k=0,1, \cdots$ and the corresponding resonance has multiplicity $2 l+1$, see Fig. 1 . The parameter $m$ denotes the mass of the black hole and $\Lambda$ is the cosmological constant: $0<9 m^{2} \Lambda<1$. Away from the zero energy, the same conclusion holds for the exact Schwarzschild metric (where we put $\Lambda=0$ ). However, as the cosmological horizon is now asymptotically flat, the meromorphic continuation of the resolvent or of the scattering matrix (and hence the definition of resonances) is more complicated.

The resonances approximated by the lattice (1.1) can be thought of as being associated to a sphere of hyperbolic orbits but the situation becomes easy due to radial symmetry. The general phenomenon of resonances associated to simple hyperbolic orbits is now well known and was studied in $[5,19,13,6,14,15]$. Ikawa [19] and Gérard [13] showed that in scattering by two strictly convex bodies the unique closed trajectory (which is hyperbolic: it is given by the ray refelected at the points of closest distance between the two bodies) generates a lattice of resonances. That seemed to constitute the first example of associating quantum objects (resonances) to hyperbolic classical dynamics. In the geometric setting of hyperbolic cylinders the same phenomenon was observed by Ch. Epstein [12] and L. Guillopé [15]. In the case of elliptic closed trajectories the construction of associated quasimodes (which correspond to eigenvalues) has a long tradition in semi-classical analysis - see for instance [10] and references given there. A

Received September, 3, 1996.

The first author is partially supported by the National Science Foundation and the Alfred P. Sloan Foundation. The second author is partially supported by the National Science Foundation and National Science and Engineering Research Council of Canada. 


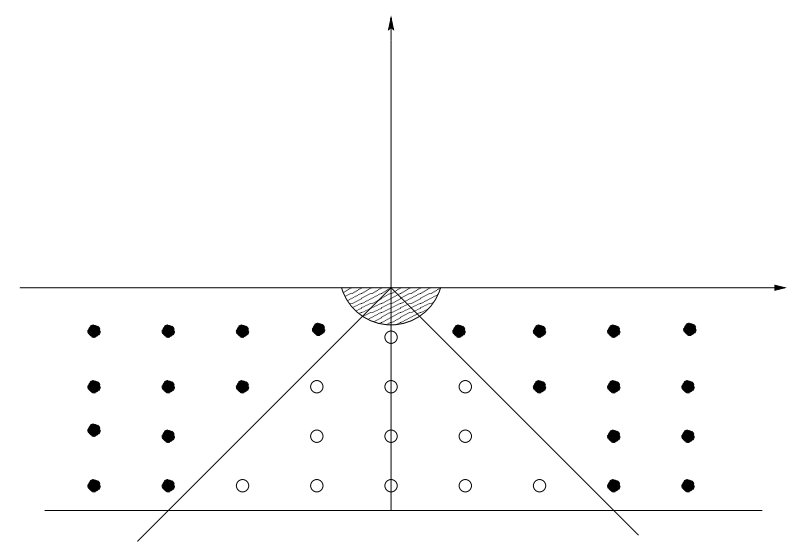

Figure 1. The lattice, $3^{-\frac{3}{2}} m\left(1-9 \Lambda m^{2}\right)^{\frac{1}{2}}\left( \pm \mathbb{N} \pm \frac{1}{2}-\frac{i}{2}\left(\mathbb{N}_{0}+\right.\right.$ $1 / 2)$ ), of pseudo-poles approximating resonances (dark dots) in a conic neighbourhood of the continuous spectrum.

formal application of the elliptic quasimode construction would produce complex eigenvalues and, as was shown by Gérard and Sjöstrand [14], that argument can be made rigorous giving semi-classical resonances.

Resonances for Black Holes correspond, according to Chandresekhar (see [8], Sect. 4.35), to its pure tones that is to the frequencies and rates of damping of signals emitted by the Black Hole in the presence of perturbations: at large times these "tones" should be a property of the Black Hole and be independent of the perturbation. The real part of a resonance corresponds to the frequency of the signal and the imaginary part to its rate of decay in time. The distance of the resonances to the real axis reflects the stability of the system under the perturbation: larger distances correspond to more stability.

From a functional analytic point of view, the scattering theory for Black Holes has been studied rigorously by Bachelot in $[2,3]$ and the existence of infinitely many resonances for the Schwarzschild model as well as some numerical computations were presented in [4]. By adding lower order terms in $l$ to the first approximation given by the pseudo-poles (1.1) the agreement with the numerical computations of Chandrasekhar-Detweiler, Leaver and Bachelot and Motet-Bachelot is quite close. The method of complex scaling used by Gérard and Sjöstrand provides also full justification for the WKB approximations of Iyer [20].

The model for an exterior of a static Black Hole is given by

$$
(Y, g), \quad Y=\mathbb{R}_{t} \times X,
$$


where in the Schwarzschild case

$$
\begin{gathered}
X=\left(r_{+}, \infty\right)_{r} \times \mathbb{S}_{\omega}^{2}, \quad g=\alpha^{2} d t^{2}-\alpha^{-2} d r^{2}-r^{2}|d \omega|^{2} \\
\alpha=\left(1-\frac{2 m}{r}\right)^{\frac{1}{2}}, \quad r_{+}=2 m
\end{gathered}
$$

and in the De Sitter-Schwarzschild case $g$ has the same form but

$$
X=\left(r_{+}, r_{++}\right)_{r} \times \mathbb{S}_{\omega}^{2}, \quad \alpha=\left(1-\frac{2 m}{r}-\frac{1}{3} \Lambda r^{2}\right)^{\frac{1}{2}}
$$

where $m>0,0<9 m^{2} \Lambda<1$ and $r_{+}, r_{++}$are the two positive roots of $\alpha=0$. We also denoted by $|d \omega|^{2}$ the standard metric on $\mathbb{S}^{2}$.

As in [8], Chapter 4 and [4] we consider the corresponding hyperbolic operator

$$
\square_{g}=\alpha^{-2}\left(D_{t}^{2}-\alpha^{2} r^{-2} D_{r}\left(r^{2} \alpha^{2}\right) D_{r}-\alpha^{2} r^{-2} \Delta_{\omega}\right),
$$

where $D_{\bullet}=\frac{1}{i} \partial_{\bullet}$ and $\Delta_{\omega}$ is the (positive) Laplacian on $\mathbb{S}^{2}$. Hence stationary scattering phenomena, and in particular resonances (which correspond to certain solutions of $\square_{g} u=0$ - see [8], Sect. 4.35), are governed by the following operator on $X$ :

$$
P=\alpha^{2} r^{-2} D_{r}\left(r^{2} \alpha^{2}\right) D_{r}+\alpha^{2} r^{-2} \Delta_{\omega} .
$$

It is shown in [4] that for $\alpha$ given in (1.3), the resolvent, $R_{X}(\lambda)=\left(P-\lambda^{2}\right)^{-1}$ has a meromorphic continuation (as an operator from $\mathcal{C}_{0}^{\infty}(\stackrel{o}{X})$ to $\mathcal{C}^{\infty}(\stackrel{o}{X})$ ) from $\Im \lambda>0$ to $\mathbb{C} \backslash i \overline{\mathbb{R}}_{-}$and below we will show that for $\alpha$ and $X$ given in (1.4), $R_{X}$ has a continuation to $\mathbb{C}$. This will be done by using the method of [22] which would also work for any perturbation of the metric preserving the behaviour at infinity (in a sense described in Sect. 3).

We then observe that after separation of variables in (1.6) and after a ReggeWheeler transformation we obtain a family of one dimensional Schrödinger operators with potentials decaying at infinity and having unique non-degenerate maxima. That constitutes the simplest example of Hamiltonians for which the classical flow has a trapped set consisiting of a single hyperbolic trajectory. Using the inverse of the angular momentum as a semi-classical parameter, the results of [14] give (1.1). Geometrically, we think of that resonant set as being associated to the sphere of closed hyperbolic trajectories described in Sect. 3.

The paper is intended as expository: we would like to show how some techniques of geometric scattering theory can be applied in a simple but interesting physical problem. It is organized as follows: In Sect. 2 we show how to apply the results of [22] in the De Sitter setting and its perturbations (only the exact Laplace-Beltrami operator was explicitly discussed in that paper) and in Sect. 3 we review the geometry of trajectories of the classical flow of $P$ and in particular the structure of the trapped set. Finally in Sect. 4 we will separate variables and apply the results of $[6,14,27,30]$ in the resulting one dimensional problem. 


\section{Meromorphic continuation}

We will now discuss the meromorphic continuation of the resolvent of $P$ given by (1.6). The poles of this continuation will then provide an elegant definition of resonances or the quasi-normal modes of [8]. The point that we would like to stress here is that only the structure of the operator at infinity (that is near $r=r_{+}$and $\left.r=r_{++}\right)$needs to be specified for this definition to make sense, that is for the meromorphic continuation to exist.

We first observe that $P$ given by (1.6) is a self-adjoint and non-negative operator with respect to the inner product given by the measure $\alpha^{-2} r^{2} d r d \omega$. Hence, by the spectral theorem,

$$
\left(P-\lambda^{2}\right)^{-1}: L^{2}\left(X ; \alpha^{-2} r^{2} d r d \omega\right) \longrightarrow L^{2}\left(X ; \alpha^{-2} r^{2} d r d \omega\right)
$$

is holomorphic for $\Im \lambda>0$. As a simple application of [22] we establish the following

Proposition 2.1. For $X$ and $\alpha$ given by (1.4) the family of operators (2.1) continues to a meromorphic family of operators in $\mathbb{C}$ :

$$
R_{X}(\lambda): \mathcal{C}_{0}^{\infty}(\stackrel{o}{X}) \longrightarrow \mathcal{C}^{\infty}(\stackrel{o}{X})
$$

with isolated poles of finite rank.

Although the proposition could be proved directly by separation of variables and a detailed analysis of the resulting one dimensional problems it is nicer to consider it as an example to which the general theory of operators on conformally compact operators applies. For that we need to review the basic assumptions and ingredients of [22].

Let $Y$ be a compact manifold with boundary given by a defining function $y$ :

$$
\partial Y=\{y=0\},\left.\quad d y\right|_{\partial Y} \neq 0,\left.\quad y\right|_{Y} ^{o}>0 .
$$

Let $g$ be a complete metric on $Y$ of the form

$$
g=\frac{h}{y^{2}}
$$

where $h$ is a $\mathcal{C}^{\infty}$ metric on $Y$ (considered as a manifold with boundary). The standard example of such a metric is provided by the hyperbolic space, $\mathbb{H}^{n}$, identified with the hyperbolic ball, $\mathbb{B}^{n}$ :

$$
\begin{gathered}
\mathbb{H}^{n} \simeq \mathbb{B}^{n}=\left\{z \in \mathbb{R}^{n}: z_{1}^{2}+\cdots+z_{n}^{2} \leq 1\right\}, \\
h=d z_{1}^{2}+\cdots+d z_{n}^{2}, \quad y=\left(1-\left(z_{1}^{2}+\cdots+z_{n}^{2}\right)\right) .
\end{gathered}
$$

The Laplacian, $\Delta_{g}$, is a second order operator which is a polynomial in vector fields vanishing at the boundary $\partial Y=\{y=0\}$. The principal part (that is that involving the highest derivatives) is in fact an elliptic polynomial in such vector 
fields (that is, a polynomial which vanishes at 0 only). In the example (2.3) but in the upper half-space model, $\mathbb{H}^{n}=\left\{(x, y): x \in \mathbb{R}^{n-1}, y \in \mathbb{R}_{+}\right\}$,

$$
\Delta_{g}=\left(y D_{y}\right)^{2}+\sum_{j=1}^{n-1}\left(y D_{x_{j}}\right)^{2}+i(n-1) y D_{y},
$$

where for $Y=\mathbb{H}^{n} y \partial_{y}$ and $y \partial_{x_{j}}$ span

$$
\mathcal{V}_{0}(Y)=\left\{V \in \mathcal{C}^{\infty}(Y ; T Y):\left.V\right|_{\partial Y} \equiv 0\right\}
$$

More generally (though it is useful to keep the metric case in mind), we can consider an arbitrary elliptic polynomial of second order with $\mathcal{C}^{\infty}$ coefficients in the elements of $\mathcal{V}_{0}(Y)$, say, $Q$. Since from the point of the standard elliptic theory (see (2.4)), $Q$ is degenerate at the boundary, the modified notion of ellipticity (in the $\mathcal{V}_{0}$-sense above) is not sufficient for the crucial step of the meromorphic continuation, namely a construction of an approximate inverse with a compact remainder for $Q-\lambda^{2}$.

The additional information is provided by the normal operator whose definition (see Sect. 2 of [22]) we will now recall. For $p \in \partial Y$ the tangent space, $T_{p} Y$, is divided into two half-spaces by the hypersurface $T_{p} \partial Y$. We will denote by $Y_{p}$ the half space on the ' $Y$ side' (that is spanned by $T_{p} \partial Y$ and the inward normal vector at $p$ ). Then any polynomial in $\mathcal{V}_{0}(Y)$ with smooth coefficients defines a natural constant coefficient operator on $Y_{p}$ :

$$
N_{p}(Q) u=\lim _{r \rightarrow 0} R_{r}^{*} f^{*} Q\left(f^{-1}\right)^{*} R_{\frac{1}{r}}^{*} u,
$$

where $u \in \mathcal{C}^{\infty}\left(Y_{p}\right), R_{r}$ is the natural $\mathbb{R}_{+}$action on $Y_{p} \simeq N_{+} T_{p} \partial Y$ given by the multiplication by $r$ on the fibres and $f$ is a local diffeomorphism from $\Omega \subset Y$, $p \in \Omega$ :

$$
f: \Omega \longrightarrow \Omega^{\prime}, \Omega^{\prime} \subset T_{p} Y, f(p)=0, d f_{p}=I, f(\partial Y) \subset T_{p} \partial Y .
$$

The definition (2.6) is independent of $f$ and although it looks quite complicated its intuitive meaning is quite clear: we freeze coefficients at a point $p$ and obtain a polynomial in the elements of $\mathcal{V}_{0}\left(Y_{p}\right)$. In the example $(2.4), N_{p}\left(\Delta_{g}\right)=\Delta_{g}$, $\mathbb{H}_{p}^{n} \simeq \mathbb{H}^{n}$ (a slightly less trivial computation with the same answer comes from considering the ball model, (2.3)).

The following proposition is implicit in [22]

Proposition 2.2. Let $Q$ be a second order differential operator on $Y$ which is a polynomial in $\mathcal{V}_{0}(Y)$ (where $\mathcal{V}_{0}(Y)$ is given by $(2.5)$ ) with coefficients in $\mathcal{C}^{\infty}(Y)$. If we assume that

(1) the principal part of $Q$ is an elliptic polynomial in the elements of $\mathcal{V}_{0}(Y)$ uniformly on $Y$, 
(2) for every $p \in \partial Y$ the normal operator of $Q$ defined by (2.6) is given by

$$
\begin{aligned}
N_{p}(Q) & =-K\left[z_{1}^{2} D_{z_{1}}^{2}+i(n-2) z_{1} D_{z_{1}}+z_{1}^{2} \sum_{i, j=2}^{n} h_{i j}(p) D_{z_{i}} D_{z_{j}}-\left(\frac{n-1}{2}\right)^{2}\right], \\
Y_{p} & =\left\{z \in \mathbb{R}^{n}: z_{1} \geq 0\right\}, \quad h_{i j} \gg 0
\end{aligned}
$$

where $K<0$ is constant on the components of $\partial Y$, then for any metric $g$ of the form $(2.2)$

$$
R_{Q}(\lambda)=\left(Q-\lambda^{2}\right)^{-1}: L^{2}\left(Y, d \operatorname{vol}_{g}\right) \longrightarrow L^{2}\left(Y, d \operatorname{vol}_{g}\right),
$$

is holomorphic for $\Im \lambda \gg 0$ and extends to a meromorphic family of operators in $\mathbb{C}$ :

$$
R_{Q}(\lambda): \mathcal{C}_{0}^{\infty}(\stackrel{o}{Y}) \longrightarrow \mathcal{C}^{\infty}(\stackrel{o}{Y})
$$

with isolated poles of finite rank.

Remark 1. In [22] only the operators of the form $\Delta_{g}$ were considered and then the meromorphy of the continuation of $\left(|d y|_{h}^{2} \Delta_{g}-s(s-n)\right)^{-1}$ from $\Re s \gg 0$ to $\mathbb{C}$ was established. The factor $-|d y|_{h}^{2}$ is equal to the asymptotic sectional curvature (all of the sectional curvatures are equal at infinity - see Lemma 2.5 in [22]) and our assumption (2) guarantees that $-|d y|_{h}^{2}=K$ is constant on the boundary components of $\partial Y$. After removing the factor $-K$ and a linear change of variables preserving the boundary of $Y_{p}$ the normal operator is the shifted Laplacian on hyperbolic space (the shift corresponds to the continuous spectrum starting at 0 so that $\lambda^{2}$ is the natural spectral parameter $): z_{1}^{2}\left(D_{z_{1}}^{2}+\right.$ $\left.\cdots D_{z_{n}}^{2}\right)+(n-2) i z_{1} D_{z_{1}}-(n-1)^{2} / 4$.

Remark 2. The structure of the poles at $\lambda \in-i(-K)^{-\frac{1}{2}} \mathbb{N}$, in particular the finiteness of their rank, needs a careful discussion as for instance in Sect. 3 of [16]. In the setting of [22] one can also follow the arguments of Sect. 5.19 of [23]. However, the corresponding resonances are not very relevant physically as they have zero energy and are far from the 'physical half-plane', $\Im \lambda>0$. We should mention however that the scattering matrix has 'non-physical' poles of infinite rank in the 'physical half-plane'. That is a well-known phenomenon observed in scattering by Yukawa type potentials and in hyperbolic scattering - see [17] for a discussion based on [22] and some references.

We will now prove Proposition 2.1, essentially by checking the assumptions of Proposition 2.2 for a slight modification of $X$ and $P$ in (1.4) and (1.6). The operator is clearly degenerate at the boundary as $\alpha\left(r_{+}\right)=\alpha\left(r_{++}\right)=0 .{ }^{1}$ Near

\footnotetext{
${ }^{1}$ For completeness, we give the values of the positive roots of $\alpha$ : $r_{+}=$ $\Im\left(\sqrt{1-(3 m \sqrt{\Lambda})^{2}}+i 3 m \sqrt{\Lambda}\right)^{\frac{1}{3}} / \sqrt{\Lambda}$ and $r_{++}=\Im\left(-\sqrt{1-(3 m \sqrt{\Lambda})^{2}}+i 3 m \sqrt{\Lambda}\right)^{\frac{1}{3}} / \sqrt{\Lambda}$
} 
each boundary component $r=r_{+}$and $r=r_{++}$we put $x=r-r_{+}$and $x=r-r_{++}$ respectively, so that the operator becomes

$$
\begin{aligned}
& \alpha_{1}(x) x D_{x}\left(\alpha_{2}(x) x\right) D_{x}+x \alpha_{1}(x) \Delta_{\omega}= \\
& \alpha_{1}(0) \alpha_{2}(0)\left(x D_{x}\right)^{2}+x \alpha_{1}(0) \Delta_{\omega}+\mathcal{O}(x)\left(x D_{x}\right)^{2}+\mathcal{O}(x) x D_{x}+\mathcal{O}\left(x^{2}\right) \Delta_{\omega}, \\
& \quad \alpha_{1}(x)=\frac{\alpha(r)^{2}}{r^{2}\left(r-r_{\bullet}\right)}, \quad \alpha_{2}(x)=\frac{r^{2} \alpha(r)^{2}}{\left(r-r_{\bullet}\right)}, \quad r_{\bullet}=r_{+}, r_{++} .
\end{aligned}
$$

To obtain an operator of the form demanded by Proposition 2.2 we change the $\mathcal{C}^{\infty}$ structure on $X$ (as a manifold with boundary) and allow a new smooth coordinate $y=x^{\frac{1}{2}}$. We will denote the new manifold by $X_{\frac{1}{2}}$ and think of $Y=X_{\frac{1}{2}}$ as a conformally compact manifold in the sense of having a metric of the form (2.2). The operator $P$ becomes

$P=\frac{1}{4} \alpha_{1}(0) \alpha_{2}(0)\left(y D_{y}\right)^{2}+\alpha_{1}(0) y^{2} \Delta_{\omega}+\mathcal{O}\left(y^{2}\right)\left(y D_{y}\right)^{2}+\mathcal{O}\left(y^{2}\right) y D_{y}+\mathcal{O}\left(y^{4}\right) \Delta_{\omega}$

and $P$ is a polynomial in the elements of $\mathcal{V}_{0}\left(X_{\frac{1}{2}}\right)$ with coefficients in $\mathcal{C}^{\infty}\left(X_{\frac{1}{2}}\right)$. To satisfy condition (2) of Proposition 2.2, that is to have the normal operator in a suitable form, we will now conjugate $P$ by a weight function:

$$
Q=((1-\chi)+\chi y) P((1-\chi)+\chi y)^{-1}
$$

where $\chi \in \mathcal{C}^{\infty}\left(X_{\frac{1}{2}} ;[0,1]\right), \chi \equiv 1$ for $y<\epsilon<1 / 2$ and $\chi \equiv 0$ for $y>2 \epsilon$. Then for any $p \in \partial X_{\frac{1}{2}}$

$$
N_{p}(Q)=\frac{1}{4} \alpha_{2}(0) \alpha_{1}(0)\left[z_{1}^{2}\left(D_{z_{1}}^{2}+\frac{4}{\alpha_{2}(0)}\left(D_{z_{2}}^{2}+D_{z_{3}}^{2}\right)\right)+i z_{1} D_{z_{1}}-1\right] .
$$

Hence we obtain the meromorphic continuation of $\left(Q-\lambda^{2}\right)^{-1}$, where $Q$ is given by (2.7). Since for $\Im \lambda \gg 0$ we have $\left(P-\lambda^{2}\right)^{-1}=((1-\chi)+\chi y)^{-1}\left(Q-\lambda^{2}\right)^{-1}((1-$ $\chi)+\chi y)$, we obtain the meromorphic continuation of $R_{X}(\lambda)$.

The resonances or quasi normal modes of the Black Hole are now defined as the poles of $R_{X}(\lambda)$ with multiplicities given as the rank of the polar part of $R_{X}(\lambda)$ (see [24] and [17]). This coincides with the definition of [8] and [4] but does not rely on the spherical symmetry and is applicable to perturbations of the metric. We remark that when the coefficients of $\square_{g}$ given here by (1.5) depend on time (as is the case for any non-stationary solution of the Einstein equations) the resonances could be defined as in the case of scattering by moving obstacles but the situation becomes more complicated, see for example [26].

\section{Geometry of classical trajectories}

In this section we study the null bicharacteristics of the operator $\square_{g}$ which is the same as studying the Hamilton flow on the non-zero energy surfaces of the operator $P$ given by (1.6). Let $p$ be the principal symbol of the operator $\square_{g}$. A curve $\gamma(s)$ in $T^{*} Y$ is said to be a bicharacteristic if it is an integral curve of the 
Hamilton vector field $H_{p}$. A curve $\gamma$ is called a null bicaracteristic if $\gamma \subset p^{-1}(0)$. The geometric significance of the bicharacteristics of $H_{p}$ is that their projection into $Y$ are geodesics of the metric $g$. The projection of the null bicharacteristics are called null geodesics. We refer the reader to [8] or [25] for a discussion on other types of bicharacteristics.

Let $(t, r, \theta, \phi, \tau, \rho, \mu, \nu)$ be local coordinates in $T^{*} Y$, where

$$
(\theta, \phi) \longmapsto(\cos \phi \cos \theta, \cos \phi \sin \theta),
$$

are the usual coordinates on $\mathbb{S}^{2}$. It follows from (1.5) that the principal symbol of $\square_{g}$ is given by

$$
\sigma_{2}\left(\square_{g}\right)=p=\alpha^{-2} \tau^{2}-\alpha^{2} \rho^{2}-r^{-2} \mu^{2}-r^{-2}(\sin \theta)^{-2} \nu^{2} .
$$

Therefore in these coordinates the Hamilton vector field of $p$ is given by

$$
\begin{gathered}
H_{p}=2 \alpha^{-2} \tau \frac{\partial}{\partial t}-2 \alpha^{2} \rho \frac{\partial}{\partial r}-2 r^{-2} \mu \frac{\partial}{\partial \theta}-2 r^{-2} \nu(\sin \theta)^{-2} \frac{\partial}{\partial \phi} \\
-\frac{\partial p}{\partial r} \frac{\partial}{\partial \rho}-2 r^{-2}(\sin \theta)^{-3} \cos \theta \nu^{2} \frac{\partial}{\partial \nu} .
\end{gathered}
$$

If $\gamma(s)$ is an integral curve of $H_{p}$ its components must satisfy

$$
\begin{gathered}
t^{\prime}(s)=2 \alpha^{-2} \tau, \quad \tau^{\prime}(s)=0, \quad r^{\prime}(s)=-2 \alpha^{2} \rho, \quad \rho^{\prime}(s)=-\frac{\partial p}{\partial r}, \quad \theta^{\prime}(s)=2 r^{-2} \mu, \\
\mu^{\prime}(s)=-2 r^{-2}(\sin \theta)^{-3} \cos \theta \nu^{2}, \quad \phi^{\prime}(s)=-2 r^{-2}(\sin \theta)^{-2} \nu, \quad \nu^{\prime}(s)=0 .
\end{gathered}
$$

Since we can always find a plane $\Pi$ containing the origin such that $(t(0), r(0), \theta(0), \phi(0)) \in \Pi$ and $(\tau(0), \rho(0), \mu(0), \nu(0))$ is tangent to $\Pi$, and since $\square_{g}$ is invariant under rotation, we can always assume that $\theta(0)=\frac{\pi}{2}$ and $\mu(0)=0$. From the uniqueness theorem for the equations in (3.1) involving $\theta$ and $\mu$ we deduce that $\theta(s)=\frac{\pi}{2}$ and $\mu(s)=0$. Hence (3.1) is reduced to

$$
\begin{gathered}
\theta(s)=\frac{\pi}{2}, \quad \mu(s)=0,2 \alpha^{2} t^{\prime}(s)=E, \quad \tau(s)=E \text { constant } \\
r^{\prime}(s)=-2 \alpha^{2} \rho, \quad \rho^{\prime}(s)=-\frac{\partial p}{\partial r}, r^{2} \phi^{\prime}(s)=L, \quad \nu(s)=-\frac{L}{2} \text { constant. }
\end{gathered}
$$

Since we are interested in the null bicharacteristics we shall also assume that $\gamma \subset p^{-1}(0)$. Therefore we have in addition

$$
\left(r^{\prime}(s)\right)^{2}+V_{L}(r)=4 E^{2} \text {, where } V_{L}(r)=r^{-2} \alpha^{2} L^{2} .
$$

Since $\left(r^{\prime}(s)\right)^{2} \geq 0$, we deduce from (3.4) that

$$
r(s) \in\left\{r: V_{L}(r) \leq 4 E^{2}\right\} .
$$




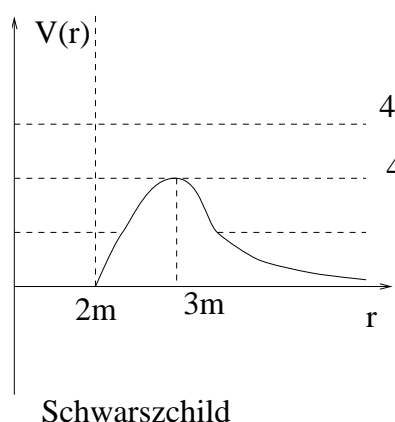

Schwarszchild

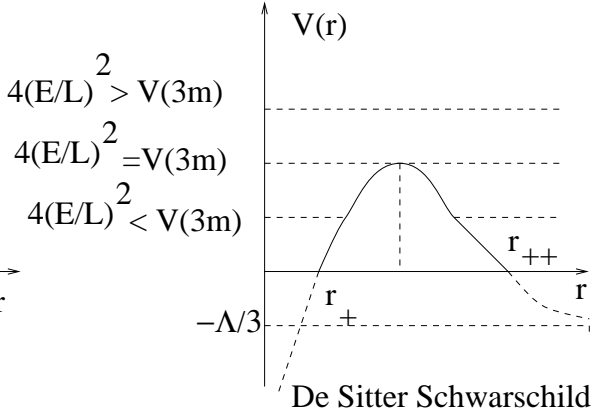

FiguRE 2. Schwarzschild and De Sitter-Schwarschild potentials.

Moreover differentiating the equation for $r^{\prime}(s)$ in (3.2) and using (3.3) we deduce that

$$
2 r^{\prime \prime}(s)=-V_{L}^{\prime}(r(s)) .
$$

It is convenient to make the potential independent of $L$. For that we consider $\phi$, instead of $s$, as a parameter. We then find that

$$
\left(\frac{1}{r(\phi)^{2}} \frac{d r}{d \phi}\right)^{2}+V(r)=4 \frac{E^{2}}{L^{2}}, \quad V(r)=r^{-2} \alpha^{2}
$$

In the two cases studied here the potential $V(r)$ is given by

$$
\begin{gathered}
\text { Schwarzschild: } V(r)=r^{-2}-2 m r^{-3}, \\
\text { De Sitter-Schwarzschild: } V(r)=r^{-2}-2 m r^{-3}-\frac{1}{3} \Lambda .
\end{gathered}
$$

The graphs of these potentials are shown in Fig. 2. These figures suggest that the only null integral curves of $H_{p}$ whose projection into $Y$ are closed occur when $V(3 m)=4 E^{2} / L^{2}$. All the other integral curves either diverge to $\infty$ or to $r=2 m$. Equation (3.6) and the graph of $V(r)$ also suggest that the orbit $r=3 m$ is unstable, that is, as the parameter $4 E^{2} / L^{2}$ approaches $V(3 m)$ the corresponding null bicaracterstics will spiral towards the circle $r=3 \mathrm{~m}$ from the inside or the outside of the circle depending on their starting points.

Next we study the type of orbit these curves project into. Let $W_{p}$ be the projection of $H_{p}$ onto $T p^{-1}(0)$. Since $H_{p}$ is tangent to $p^{-1}(0), W_{p}$ is the restriction of $H_{p}$ to $p^{-1}(0)$. The main result of this section is

Proposition 3.1. Let $\tilde{\gamma}$ be a null bicharacteristic contained in $\left\{\theta=\frac{\pi}{2}, \mu=0\right\}$. Let $\gamma$ be its projection to $X$. If $\gamma$ is a closed curve then $\tilde{\gamma}$ is given by

$$
r(s)=3 m, \quad \rho(s)=0, \quad t(s)=t_{0}+6 E s, \quad \tau(\phi)=E, \quad \nu(s)=-\frac{L}{2} .
$$

Moreover $\gamma$ is a closed hyperbolic trajectory of the vector field $V_{p}$ obtained by projecting $W_{p}$ into $T\left(X \cap\left\{\theta=\frac{\pi}{2}\right\}\right)$. 
Proof. Suppose that $\gamma$ is a closed trajectory. Since $H_{p}$ is invariant under the rotations $\phi \mapsto \phi+c, \gamma$ must be a circle. Therefore $r^{\prime}(s)=0$ and it follows from (3.5) that $V^{\prime}(r(s))=0$. Thus $r(s)=3 \mathrm{~m}$. The remaining equations in (3.7) follow directly from (3.2).

To prove that $\gamma$ is a closed hyperbolic trajectory of $V_{p}$ we use the geometric interpretation of the null bicharacteristics. We deduce that the integral curves of $V_{p}$ are geodesics of the restriction of the metric $g$ to $X \cap\left\{\theta=\frac{\pi}{2}\right\}$. In local coordinates the restriction of $g$ to this submanifold is given by

$$
h=\alpha^{-2} d r^{2}+r^{2} d \phi^{2} .
$$

The curvature of this metric is given by $K(r)=r \alpha \frac{d}{d r}\left(r^{-2} \alpha\right)$. Hence in the two cases we find

$$
\begin{gathered}
\text { Schwarzschild : } K_{S}(r)=r^{-3}(5 m-2 r) \\
\text { Schwarzschild-De Sitter: } K_{S D}(r)=r^{-2}\left(\frac{5 m}{r}-2+\frac{\Lambda r^{2}}{3}\right) .
\end{gathered}
$$

When $r=3 m$ we find

$$
K_{S}(r)=-m(3 m)^{-3}<0, \quad K_{S D}(r)=\frac{1}{3}(3 m)^{-2}\left(9 m^{2} \Lambda-1\right)<0 .
$$

Thus for $r-3 m$ small enough we deduce that the curvature $K(r)$ is negative in both the Schwarzschild and Schwarzschild-De Sitter metrics and this implies that $\gamma$ is a hyperbolic orbit.

It follows directly from Proposition 3.1 that

Proposition 3.2. The sphere $\mathbb{S}_{h}=\{(r, \omega) \in X: r=3 m\}$ is the union of closed geodesics of $g^{\sharp}=\alpha^{-2} d r^{2}+r^{2}|d \omega|^{2}$. Moreover each one of these curves is planar and is a hyperbolic geodesic of the metric $g^{\sharp}$ restricted to the plane containing it.

We note that the weak hyperbolicity of $\gamma$ means that the eigenvalues of its Poincaré map are given by $1,1, k, k^{-1}$, where $k>1$.

\section{Resonances associated to the family of closed hyperbolic trajectories}

Since there are no ready-to-use theorems on resonances associated to manifolds of (degenerate) hyperbolic trajectories on non-euclidean (in this case hyperbolic-like) spaces, we will exploit the spherical symmetry to reduce our problem to a semi-classical problem on the line. Nevertheless, we would like to keep the analysis of Sect. 3 in mind as it provides the underlying geometry.

Let $P$ be the operator defined in (1.6) and let

$$
\widetilde{P}=r P r^{-1} .
$$


We find that

$$
\widetilde{P}=\alpha^{2} D_{r}\left(\alpha^{2} D_{r}\right)+\alpha^{2} r^{-2} \Delta_{\omega}+r^{-1} \alpha^{2}\left(\partial_{r} \alpha^{2}\right) .
$$

Let $x=x(r)$ be defined as

$$
x^{\prime}(r)=\alpha^{-2} .
$$

In coordinates $(x, \omega)$ the operator $\widetilde{P}$ is given by

$$
\widetilde{P}=D_{x}^{2}+\alpha^{2} r^{-2} \Delta_{\omega}+\alpha^{2} r^{-1}\left(\partial_{r} \alpha^{2}\right), \text { where } r=r(x) .
$$

A function

$$
u(x, \omega)=\sum_{l, m} a_{l, m}(x) Y_{l}^{m}(\omega),
$$

$$
\text { where } \Delta_{\omega} Y_{l}^{m}(\Omega)=l(l+1) Y_{l}^{m}(\omega)
$$

is a generalized eigenfunction of $\widetilde{P}$ with eigenvalue $\lambda^{2}$ if and only if

$$
\begin{gathered}
\left(D_{x}^{2}+V_{l}(x)\right) a_{l, m}=\lambda^{2} a_{l, m}, \\
\text { with } V_{l}(x)=\alpha^{2} r^{-2}\left(l(l+1)+r \partial_{r}\left(\alpha^{2}\right)\right) .
\end{gathered}
$$

The equation (4.4) can be transformed into a semi-classical one:

$$
\begin{gathered}
(P(x, h D ; h)-z) u=0, \quad P(x, h D ; h)=\left(h D_{x}\right)^{2}+V(x, h), \\
V(x, h)=\alpha^{2} r^{-2}\left(1+h^{2} r \partial_{r}\left(\alpha^{2}\right)\right), \quad h=(l(l+1))^{-\frac{1}{2}}, \quad z=h^{2} \lambda^{2} .
\end{gathered}
$$

The resolvents $(P(x, h D ; h)-z)^{-1}, 0<\arg z<2 \pi$, and $\left(P-\lambda^{2}\right)^{-1}, \Im \lambda>0$ can be expressed in terms of each other using (4.1)-(4.5) and the same is true for their meromorphic continuations through the continuous spectrum $\arg z=0$ and $\Im \lambda=0$ respectively. The meromorphy of $(P(x, h D ; h)-z)^{-1}: \mathcal{C}_{0}^{\infty}(\mathbb{R}) \rightarrow \mathcal{C}^{\infty}(\mathbb{R})$ for $z \in \Lambda \cap\{-\theta<\arg z<2 \pi\}$ for $\theta$ small enough, can however be seen directly by the method of complex scaling (see [1] for the original mathematical treatment and [14] for more recent references - here we will follow the exposition of [18] and [29]).

We start with a description of the holomorhic continuation of the potential:

Proposition 4.1. The potential in (4.5) extends to a holomorphic function in a conic neighbourhood of the real axis given by $|\arg w|<\varphi$ and moreover it satisfies

$$
|V(w, h)| \leq\left\{\begin{array}{lll}
C \exp (-|w| / C) & \alpha \text { given by }(1.3) & \Re w \rightarrow-\infty \\
1 /|w|^{2} & \alpha \text { given by }(1.3) & \Re w \rightarrow \infty \\
C \exp (-|w| / C) & \alpha \text { given by }(1.4) & \Re w \rightarrow \pm \infty
\end{array}\right.
$$


Proof. We shall prove the case where $\alpha$ is given by (1.3). The Schwarzschild-De Sitter case is a little more technical, but follows from similar arguments and will be left to the reader.

In this case

$$
x(r)=r+2 m \log (r-2 m), \quad r>2 m .
$$

Thus $x(r)$ is a strictly increasing real analytic function of $r$, hence it has a real analytic inverse $r(x)$. We would like to extend $r(x)$ holomorphically to a conic neighbourhood $\Gamma$ of the real axis.

As in Proposition IV.2 of [4], it follows from the Lagrange inversion formula, see [11], that there exist $A>0$ such that $r(x)$ has a holomorphic extension to the region $\{w \in \mathbb{C}:|\Re w|>A\}$. Since $r(x)$ is real analytic in $\mathbb{R}$, one can extend it holomorphically to a region $\{w \in \mathbb{C}:|\Im w|<\epsilon,|\Re w|<A\}$. Unique continuation gives a holomorphic extension of $r(x)$ to a conic neighborhood of the real axis. The bounds in (4.6) follow exactly as in [4].

We can now consider the differential operator with holomorphic coefficients,

$$
P\left(w, h D_{w} ; h\right),
$$

and then define the scaled operator:

$$
P_{\theta}\left(x, h D_{x} ; h\right)=\left.P\left(w, h D_{w} ; h\right)\right|_{\Gamma_{\theta}}, \quad \Gamma_{\theta}=e^{i \theta} \mathbb{R},
$$

where we parametrize $\Gamma_{\theta}$ by $x, w=e^{i \theta} x$. The scaled operator is not selfadjoint but its $L^{2}$-eigenvalues coincide with the resonances defined as poles of the meromorphic continuation of the resolvent. This is made precise by the following

Proposition 4.2. For $-2 \theta<\arg z<2 \pi-2 \theta$ the operator

$$
P_{\theta}(x, h D ; h)-z: H^{2}\left(\Gamma_{\theta}\right) \longrightarrow L^{2}\left(\Gamma_{\theta}\right),
$$

is a Fredholm operator of index 0 . The eigenvalues of $P_{\theta}(x, h D ; h)$ in $-2 \theta<$ $\arg z<2 \pi-2 \theta$ coincide with the poles of of the meromorphic continuation of $(P(x, h D ; h)-z)^{-1}$ there.

Before outlining the ideas behind the proof we remark that in one dimension all the eigenvalues and consequently the resonances are simple.

The reason for the Fredholm property of the scaled operator lies in the simple analysis of the free operator, $\left(h D_{x}\right)^{2}$. Its scaled version is given by $e^{-2 i \theta}\left(h D_{x}\right)^{2}$, so that if $-2 \theta<\arg z<2 \pi-2 \theta$ then $e^{-2 i \theta} D_{x}^{2}-z$ is in fact invertible on $L^{2}$. To study the operator $P_{\theta}(x, h D ; h)-z$ we can consider it as an elliptic operator on compact subsets of $\Gamma_{\theta}$ and then construct its local approximate inverse there (in the semi-classical sense, that is modulo $\mathcal{O}\left(h^{\infty}\right)$ and smoothing operators). Near infinity, the free part, $e^{-i 2 \theta} D_{x}^{2}-z$ dominates in view of (4.6) and hence from its invertibility we can get an approximate inverse near infinity. By gluing the two constructions we obtain an approximate inverse modulo compact operators 
which in turn implies that the operator $P_{\theta}(x, h D ; h)-z$ is Fredholm with index zero.

To see that the poles of the meromorphic continuation of $(P(x, h D ; h)-z)^{-1}$ and those of the meromorphic operator $\left(P_{\theta}(x, h D ; h)-z\right)^{-1}: L^{2}\left(\Gamma_{\theta}\right) \rightarrow L^{2}\left(\Gamma_{\theta}\right)$ (that is the eigenvalues of $P_{\theta}$ ) coincide, we introduce a new space of functions, $\mathcal{A}_{\theta}$ consisting of functions holomorphic in a neighbourhood of the region $-\theta-\epsilon<$ $\arg ( \pm w)<\theta+\epsilon$ and such that for $u \in \mathcal{A}_{\theta},|u(w)| \leq C \exp \left(-C|w|^{2}\right)$ there. We note that the set $\left\{\left.u\right|_{\Gamma_{\theta^{\prime}}}: u \in \mathcal{A}_{\theta}\right\}, 0<\theta^{\prime}<\theta$, is dense in $L^{2}\left(\Gamma_{\theta^{\prime}}\right)$ and it can be shown that $(P(x, h D ; h)-z)^{-1}$ continues meromorphically as an operator from $\left\{\left.u\right|_{\mathbb{R}}: u \in \mathcal{A}_{\theta}\right\}$ to $\mathcal{C}^{\infty}(\mathbb{R})$ with poles coinciding with the poles of the continuation on $\mathcal{C}_{0}^{\infty}(\mathbb{R})$ (since the continuation is really that of the distributional Schwartz kernel of the operator, this is quite clear). Now, if $v \in \mathcal{A}_{\theta}$ then, provided that $z$ is not in the spectrum of $P_{\theta}(x, h D ; h)$ and that $-2 \theta<\arg z<2 \pi-2 \theta$ we have the existence of $u_{\theta}=\left.\left(P_{\theta}(x, h D ; h)-z\right)^{-1} v\right|_{\Gamma_{\theta}}$. Since $u_{\theta}$ solves an ordinary differential equation with holomorphic coefficients, it extends to a holomorphic function in a neighbourhood of $\Gamma_{\theta}$ and in fact we obtain a function $u$ holomorphic in $-\theta<\arg ( \pm w)<\theta$ such that $u_{\theta}=\left.u\right|_{\Gamma_{\theta}}$ and that $\left(P\left(w, h D_{w} ; h\right)-z\right) u=v$. Hence we also have

$$
\left.u\right|_{\mathbb{R}}=(P(x, h D ; h)-z)\left(\left.v\right|_{\mathbb{R}}\right)
$$

which gives an expression for the meromorphic continuation of $(P(x, h D ; h)-$ $z)^{-1}$ on $\mathcal{A}_{\theta}$ to $\arg z>-2 \theta$. The poles coincide with those of $\left(P_{\theta}(x, h D ; h)-z\right)^{-1}$.

The special property of the potential $V(x, h)$ in $(4.5)$ is the existence of a unique non-degenerate maximum. This is clear from Fig. 2, which shows its leading semi-classical part $V(x, 0)$. We can also easily compute that for the leading semi-classical term in the potential, $V_{0}(x)=V(x, 0): V_{0}^{\prime}(x)=0$ only for $x=x_{0}$ where $r\left(x_{0}\right)=3 m$ and that

$$
\begin{aligned}
& V_{0}^{\prime \prime}\left(x_{0}\right)= \\
& \left.\quad\left(\frac{d x}{d r}\right)^{-2} \frac{d^{2} V_{0}}{d r^{2}}\right|_{r=3 m}=\left.\alpha^{4} \frac{d^{2}}{d r^{2}}\left(\alpha^{2} r^{-2}\right)\right|_{r=3 m}=-2 \cdot 3^{-6}\left(1-9 \Lambda m^{2}\right)^{2} m^{-4} .
\end{aligned}
$$

From the dynamical point of view this means that the flow of the Hamiltonian given by the principal symbol of $P(x, h D ; h), \quad p(x, \xi)=\xi^{2}+V_{0}(x)$, has an unstable equilibrium point at $\left(x_{0}, 0\right)$. Hence, we can apply the general result of Theorem 1 in [14] on resonances associated to a closed hyperbolic trajectory and that gives

Proposition 4.3. For any $C_{0}>0$ and any $a<V\left(x_{0}\right), b>0$ let

$$
\Gamma_{0}(h)=\left\{V_{0}\left(x_{0}\right)-i h\left(\frac{1}{2}\left|V_{0}^{\prime \prime}\left(x_{0}\right)\right|\right)^{\frac{1}{2}}(2 k+1): k=0,1,2, \cdots\right\} .
$$




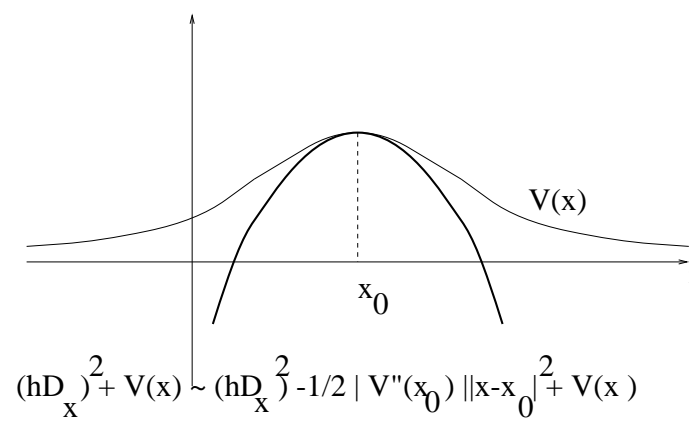

FiguRE 3. Quadratic approximation near unstable equilibrium.

Then for $h$ small enough there exists an injective map $b(h)$ from $\Gamma_{0}(h)$ into the set of resonances of $P(x, h D ; h)$ such that

$$
b(h)(\mu)-\mu=o(h), h \longrightarrow 0, \quad \mu \in \Gamma_{0}(h),
$$

and such that all resonances in

$$
\left[V_{0}\left(x_{0}\right)-a, V\left(x_{0}\right)+b\right]-i\left[0, C_{1} h\right]
$$

are in the image of $b(h)$. Here $C_{1}, 0<C_{1}<C_{0}$, is a fixed arbitrary constant.

We should stress that this is a degenerate, one dimensional form of Theorem 1 in [14] and it really belongs to the results of [27]. In dimension one it could probably be improved to cover a larger region in $\mathbb{C}$. Also, the result is uniform with respect to $b$ : once the real part is large then there are no resonances in the corresponding strip (in fact, a conic neighbourhood of $\mathbb{R}$ - see [6] and [28]).

To indicate the idea of the proof we will approximate the potential by its quadratic part near the maximum (see Fig. 3): in the semi-classical régime the contribution of the potential is localized once the behaviour at infinity is controlled (which is done using the complex scaling method described after the statement of Proposition 4.3). Hence, let us consider the approximation

$$
P^{\sharp}(x, h D ; h)=\left(h D_{x}\right)^{2}-\frac{1}{2}\left|V_{0}^{\prime \prime}\left(x_{0}\right)\right|\left(x-x_{0}\right)^{2}+V\left(x_{0}\right)
$$

to which we can apply complex scaling formally:

$$
P_{\theta}^{\sharp}(x, h D ; h)=\left.P^{\sharp}\left(w, h D_{w} ; h\right)\right|_{\Gamma_{\theta}^{\sharp}}, \Gamma_{\theta}^{\sharp}=x_{0}+e^{i \theta} \mathbb{R} \subset \mathbb{C},
$$

so that with the coordinate $y$ on $\Gamma_{\theta}^{\sharp}, w=x_{0}+e^{i \theta} y$,

$$
P_{\theta}^{\sharp}\left(y, h D_{y} ; h\right)=e^{-2 i \theta}\left(h D_{y}\right)^{2}-\frac{1}{2} e^{2 i \theta}\left|V^{\prime \prime}\left(x_{0}\right)\right| y^{2}+V\left(x_{0}\right) .
$$

Setting $\theta=\frac{\pi}{4}$ we effectively turn our operator into a multiple of the harmonic oscillator

$$
P_{\frac{\pi}{4}}^{\sharp}\left(y, h D_{y} ; h\right)=-i\left(\left(h D_{y}\right)^{2}+\frac{1}{2}\left|V^{\prime \prime}\left(x_{0}\right)\right| y^{2}\right)+V\left(x_{0}\right) .
$$




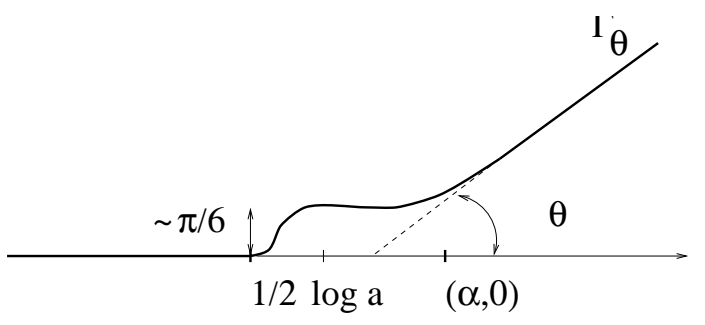

Figure 4. The $\alpha$-dependent complex scaling.

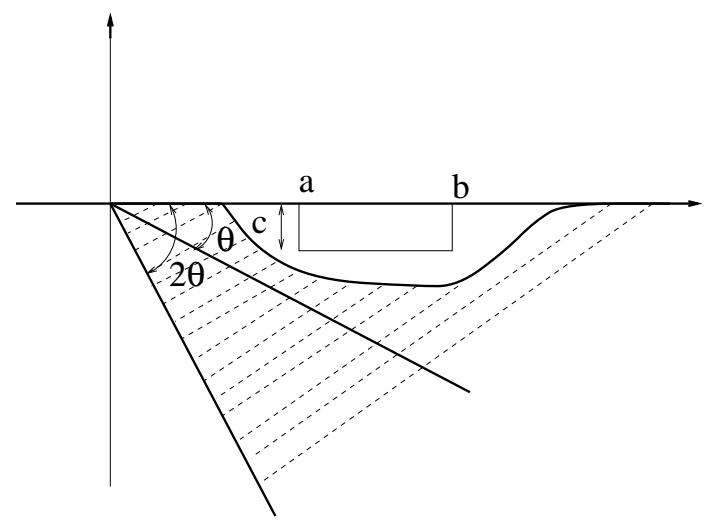

Figure 5. The values taken by $\rho^{2}+\left.e^{-2 r} a\left(\alpha, e^{-r}\right)\right|_{T^{*} \Gamma_{\theta}}$.

Since the eigenvalues of the harmonic oscillator are $\left(h D_{y}\right)^{2}+a y^{2}$ are given by $a^{\frac{1}{2}} h(2 k+1), k=0,1, \cdots$ we see that the eigenvalues of $P_{\frac{\pi}{4}}^{\sharp}$ in the rectangle around $V_{0}\left(x_{0}\right)$ are given by the elements of the set $\Gamma^{0}(h)$ (We should mention that a less accurate result than the proposition quoted above, was obtained by Briet, Combes and Duclos in [7] and the proof is based on the outline recalled above).

To apply Proposition 4.2 to the Black Hole problem we need a better uniformity in $l$ : more precisely we need to obtain the absence of resonances of $P(x, h D ; h)$ in a rectangle $[K h, \epsilon]-i[0, C h]$. Then in the rescaling to the original problem, as described in (4.4)-(4.5) we obtain a good approximation of the resonances by the lattice of pseudo-poles (1.1) outside a fixed neighbourhood of the origin (that is of the bottom of the continuous spectrum).

The study of resonance free regions is based on lower bounds for the scaled operator $P_{\theta}-z$. To obtain uniformity in those bounds as $x \rightarrow-\infty$ for the Schwarzschild and as $|x| \rightarrow \infty$ for the De Sitter-Schwarzschild metric we have to use a modified version of complex scaling from [30]. We have the following general proposition:

Proposition 4.4. Let $Q(\alpha, \tilde{h})$ be a family of second order differential operators 
on $[1, \infty)$ defined using the Dirichlet boundary condition and of the form

$$
Q(\alpha, \tilde{h})=\left(h D_{r}\right)^{2}+e^{-2 r} a(\alpha, r),
$$

where $\alpha \in[1, \infty)$ and $a(\alpha, r)$ is holomorphic in $r$ for $r$ in a conic neighbourhood of the positive real axis. We assume that a is elliptic in the sense that

$$
\alpha^{2} / C \leq a(\alpha, x) \leq C \alpha^{2},
$$

and $C>1$ is independent of $r$ and that it has a limit at infinity:

$$
\frac{a(\alpha, r)}{\alpha^{2}} \longrightarrow \frac{a_{0}(\alpha)}{\alpha^{2}} \quad \text { as } r \longrightarrow \infty,|\arg (r)|<\gamma_{0}
$$

Then for $0<\theta<\theta_{0}, 0<\tilde{h}<\tilde{h}_{0}$ and $\alpha>\alpha_{0}$ the meromorphic continuation of $(Q(\alpha, \tilde{h})-z)^{-1}$ for $\Im z>0$ has no poles in

$$
R_{\theta}=\{z: a \leq \Re z \leq b,|\Im z|<c\} \cap\{|\arg z|<\theta\},
$$

where $a<b$ and $c$ are fixed constants independent of $\tilde{h}$ and $\theta$.

We used a different notation for the semi-classical parameter, $\tilde{h}$, now as it is going to be different than in (4.5).

The proof of Proposition 4.4 depends only on analyticity and the behaviour at infinity. The new scaling (see Fig. 4) gives an operator for which one can find uniform lower bounds. More precisely, the subset of $\mathbb{C}$ avoided by the values of the symbol of the scaled operator (see Fig. 5) does not contain the poles of $(Q(\alpha, \tilde{h})-z)^{-1}$ if $\tilde{h}$ is small enough. Applying this in the De Sitter-Schwarzschild case where the potential is exponentially decaying at both infinities and $\alpha^{2}=$ $l(l+1) \tilde{h}^{2}$, gives the absence of resonances of the operator (4.4) in the region

$$
\left\{\frac{a}{h_{0}^{2}}<\Re z<\frac{b l(l+1)}{\alpha_{0}^{2}}\right\} \cap\{\arg z>-\beta\}, \quad l(l+1)>\left(\frac{\alpha_{0}}{h_{0}}\right)^{2},
$$

for $\beta>0$ small enough.

For the potential (4.4) arising from the Schwarzschild metric (1.3) we have to use the modified scaling on the left, that is as $x \rightarrow-\infty$ and the usual scaling as $x \rightarrow+\infty$ (see Fig. 6). For $x$ positive the potential behaves as $C x^{-2}$ and we can use the argument from [6] for resonance free regions near thresholds: for $z$ in $\left\{\Im z>-\Re z / C+\mathcal{O}\left(h^{2}\right),|z| \leq C\right\}$ and for functions, $u \in \mathcal{C}_{0}^{\infty}(\Gamma)$, supported in $\Gamma_{\theta} \cap\{\Re w>-1\}$ we have a uniform bound

$$
\left\|\left(P_{\theta}(x, h D ; h)-z\right) u\right\| \geq\left(\Re z-\mathcal{O}\left(h^{2}\right)\right)\|u\| / C^{\prime},
$$

where $P_{\theta}$ is given by (4.7). Rescaling to the $l$-dependent situation (4.4) and using the method of Proposition 4.4 in $\Gamma_{\theta} \cap\{\Re w<0\}$ we obtain the same resonance free region (4.9) for the case (1.3) (that is, the same for the Schwarzschild case as for the De Sitter-Schwarzschild case).

Finally, we recall that since the potentials here are analytic, for any fixed $h$ there are only finitely many resonances in $\{\arg z>-\theta\} \cap\{\Re z>\epsilon\}$ for any small 


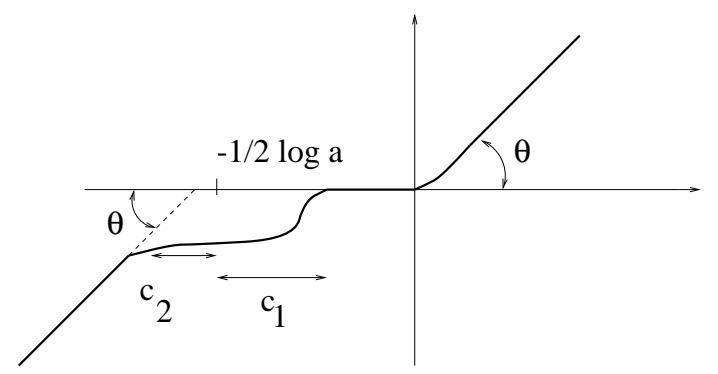

FiguRE 6. Scaling in the Schwarzschild case.

$\theta$ and any $\epsilon$ (see [1] and [28]). Combining this with Proposition 4.3, the discussion following Proposition 4.4 and using the relations $z=\lambda^{2} h^{2}, h=[l(l+1)]^{-\frac{1}{2}}$, we obtain, uniformly in any strip,

$$
\lambda^{2}=\frac{\left(1-9 \Lambda m^{2}\right)}{3^{3} m^{2}} l(l+1)\left(1-i[l(l+1)]^{-\frac{1}{2}}(2 k+1)\right),
$$

Expansions of the square roots for large values of $l$ and the symmetry with respect to the imaginary axis now give

Theorem. Let $P$ be given by (1.6) with $\alpha$ given by either (1.3) or (1.4). Then, there exists $K>0$ and $\theta>0$ such that for any $C>0$ there exists an injective map, $\tilde{b}$, from the set of pseudo-poles

$$
\frac{\left(1-9 \Lambda m^{2}\right)^{\frac{1}{2}}}{3^{\frac{3}{2}} m}\left( \pm \mathbb{N} \pm \frac{1}{2}-i\left(\mathbb{N}_{0}+\frac{1}{2}\right)\right)
$$

into the set of poles of the meromorphic continuation of $\left(P-\lambda^{2}\right)^{-1}$ such that all the poles in

$$
\Omega_{C}=\{\lambda: \Im \lambda>-C,|\lambda|>K, \Im \lambda>-\theta|\Re \lambda|\}
$$

are in the image of $\tilde{b}$ and for $\tilde{b}(\mu) \in \Omega_{C}$,

$$
\tilde{b}(\mu)-\mu \longrightarrow 0 \text { as }|\mu| \rightarrow \infty \text {. }
$$

If $\Re \mu=3^{-\frac{3}{2}} m^{-1}\left(1-9 \Lambda m^{2}\right)^{\frac{1}{2}}( \pm l \pm 1 / 2), l=1,2, \cdots$, then the corresponding pole, $\tilde{b}(\mu)$, has multiplicity $2 l+1$.

The difference between the Schwarzschild and the De Sitter-Schwarzschild cases is exhibited by the behaviour near $\lambda=0$. In the latter case Proposition 2.1 guaranteed a global meromorphic continuation with isolated poles of finite rank. Hence a fixed neighbourhood of the origin can contain at most finitely many poles and the theorem above describes all the poles of $\left(P-\lambda^{2}\right)^{-1}$ in strips, except possibly finitely many.

In the Schwarzschild case the complex scaling method as applied in [4] gives the meromorphy of $\left(P-\lambda^{2}\right)^{-1}$ in $\mathbb{C} \backslash \overline{\mathbb{R}}$ only. Hence an accumulation of poles at the origin cannot be automatically excluded. A more detailed analysis of 
the Regge-Wheeler potential (4.4) could perhaps show that such behaviour is impossible.

\section{Acknowledgement}

We would like to thank A. Bachelot for suggesting the De Sitter model as particularly suitable for the general methods of geometric scattering theory.

\section{References}

1. J. Aguilar and J.-M. Combes, A class of analytic perturbations for one-body Schrödinger Hamiltonians, Comm. Math. Phys. 22 (1971), 269-279.

2. A. Bachelot, Gravitational scattering of electromagnetic field by Schwarzschild black hole, Ann. Inst. H. Poincaré, Phys. Théor. 54 (1991), 261-320.

3. __ Scattering of electromagnetic field by De Sitter-Schwarzschild black hole, in Nonlinear hyperbolic equations and field theory, Pitman Res. Notes Math. Ser. 253 (1992), 23-35.

4. A. Bachelot and A. Motet-Bachelot, Les résonances d'un trou noir de Schwarzschild, Ann. Inst. H. Poincaré, Phys. Théor. 59 (1993), 3-68.

5. C. Bardos, J.-C. Guillot, and J.V. Ralston, La relation de Poisson pour l'équation des ondes dans un ouvert non borné, Comm. Partial Differential Equations 7 (1982), 905-958.

6. P. Briet, J.-M. Combes, and P. Duclos., On the location of resonances in the semi-classical limit I. Resonance free domains, J. Math. Anal. Appl. 126 (1987), 90-99.

7. ferential Equations 12 (1987), 201-222.

8. S. Chandrasekhar, The mathematical theory of black holes, Oxford University Press, New York, 1983.

9. S. Chandrasekhar and S. Detweiler, The quasi-normal modes of the Schwarzschild blackhole, Proc. Roy. Soc. London Ser. A 344 (1975), 441-452.

10. Y. Colin de Vedière, Quasimodes sur les variétés riemannienes, Invent. Math. 43 (1977), $15-52$.

11. N. G. De Bruijn, Asymptotic methods in analysis, Dover, New York, 1981.

12. Ch. Epstein, unpublished.

13. Ch. Gérard, Asymptotique des pôles de la matrice de scattering pour deux obstacles strictement convexes, Bull. Soc. Math. France 116 (1988).

14. Ch. Gérard and J. Sjöstrand, Semiclassical resonances generated by a closed trajectory of hyperbolic type, Comm. Math. Phys. 108 (1987), 391-421.

15. L. Guillopé. Sur la distribution de longuers des géodésique fermés s'une surface compact $\grave{a}$ bord totalement geodesique, Duke Math. J. 53 (1986), 827-848.

16. L. Guillopé and M. Zworski. Polynomial bounds on the number of resonances for some complete spaces of constant negative curvature near infinity, Asymptotic Anal. 11 (1995), $1-22$.

17. Scattering asymptotics for Riemann surfaces, Ann. of Math. (to appear).

18. W. Hunziker, Distortion analyticity and molecular resonance curves, Ann. Inst. H. Poincaré. Phys. Théor. 45 (1986), 339-358.

19. M. Ikawa, On the poles of the scattering matrix for two convex obstacles, J. Math. Kyoto Univ. 23 (1983), 127-194.

20. S. Iyer, Black hole normal modes: a WKB approach II; Schwarzschild black holes, Phys. Rev. D 35 (1987), 3632-3636.

21. E. Leaver, Spectral decomposition of the perturbation response of the Schwarzschild geometry, Phys. Rev. D 34 (1986), 384-408. 
22. R. R. Mazzeo and R. B. Melrose, Meromorphic continuation of the resolvent on complete spaces with asymptotically constant negative curvature, J. Funct. Anal. 75 (1987), 260-310.

23. R. B. Melrose, The Atiyah-Patodi-Singer index theorem, Res. Notes Math., A K Peters, Wellesley, 1993.

24. _ Geometric scattering theory, Cambridge University Press, Cambridge, New York, Melbourne, 1995.

25. B. O'Neill, Semi-Riemannian geometry with applications to relativity, Academic Press, 1983.

26. V. Petkov, Scattering theory for hyperbolic operators, Stud. Math. Appl., 21, NorthHolland Publ. Co., Amsterdam, 1989.

27. J. Sjöstrand, Semiclassical resonances generated by non-degenerate critical points, in Pseudo-differential operators., ed. H.O. Cordes et al, Lecture Notes in Math. 1256, Springer Verlag, 1986.

28. , Geometric bounds on the density of resonances for semi-classical problems, Duke Math. J. 60 (1990), 1-57.

29. J. Sjöstrand and M. Zworski, Complex scaling and the distribution of scattering poles. J. Amer. Math. Soc. 4 (1991), 729-769.

30. M. Zworski, Dimension of the limit set and the density of resonances for convex cocompact hyperbolic quotients, preprint, 1996.

Deptartment of Mathematics, Purdue University, West Lafayette, IN 47907-1395 E-mail address: sabarre@math.purdue.edu

Department of Mathematics, University of Toronto, Toronto, Ontario, CANAdA M5S 1A4

E-mail address: zworski@math.toronto.edu 